To appear in the Journal of Difference Equations and Applications Vol. 00, No. 00, April 2016, 1-14

\title{
Remarks on homoclinic structure in Bogdanov-Takens map
}

\author{
B. Al-Hdaibat ${ }^{a}$, W. Govaerts ${ }^{b *}$, D.L. van Kekem ${ }^{c}$, and Yu.A. Kuznetsov ${ }^{d, e}$ \\ ${ }^{a}$ Department of Mathematics, Hashemite University, Zarqa 13115, Jordan \\ ${ }^{b}$ Department of Applied Mathematics, Computer Science and Statistics, Ghent \\ University, Krijgslaan 281-S9, B-9000 Gent. \\ ${ }^{c}$ Johann Bernoulli Institute for Mathematics and Computer Science, University of \\ Groningen, P.O. Box 407, 9700 AK Groningen, The Netherlands. \\ ${ }^{d}$ Department of Mathematics, Utrecht University, P.O. Box 80010, \\ 3508 TA Utrecht, The Netherlands \\ e Department of Applied Mathematics, University of Twente, P.O. Box 217, \\ 7500 AE Enschede, The Netherlands
}

(December 2015)

\begin{abstract}
It is known that in the Bogdanov-Takens map there exists a zone of transversal homoclinic intersections bounded by two curves of homoclinic tangencies. In this paper, we derive an improved asymptotic formula for the homoclinic parameter values of the BT map. We compare two methods to approximate the Bogdanov-Takens map by the time-1 flow of a vector-field, and find that they are equivalent. We show that it is essential to include the second-order terms w.r.t. the parameters to obtain a more accurate asymptotic for the homoclinic zone. We show how to use this new homoclinic asymptotic to compute branches of homoclinic tangencies in the BT map numerically, obtaining the whole homoclinic structure of the Bogdanov-Takens map.
\end{abstract}

Keywords: Bogdanov-Takens map; homoclinic intersections; homoclinic asymptotic; MatContM

AMS Subject Classification: 37C05; 37G20; 37G25; 65P20; 65P30

\section{Introduction}

The Bogdanov-Takens (BT) bifurcation plays an important role in the study of dynamical systems since it implies a global (homoclinic) bifurcation [2. Improved asymptotics of the homoclinic bifurcation in a neighborhood of a BT bifurcation point for vector-fields have been obtained recently [1, 17]. In the present paper we discuss the homoclinic structure in the two-parameter map

$$
G(u, \nu):\left(\begin{array}{l}
u_{0} \\
u_{1}
\end{array}\right) \mapsto\left(\begin{array}{ll}
1 & 1 \\
0 & 1
\end{array}\right)\left(\begin{array}{l}
u_{0} \\
u_{1}
\end{array}\right)+\left(\begin{array}{c}
0 \\
\nu_{1}+\nu_{2} u_{1}+a u_{0}^{2}+b u_{0} u_{1}
\end{array}\right)
$$

where $u=\left(u_{0}, u_{1}\right) \in \mathbb{R}^{2}, \nu=\left(\nu_{1}, \nu_{2}\right) \in \mathbb{R}^{2}$ and $(a, b) \in \mathbb{R}^{2}$. The map (1) (or simply the $B T$ map) is a truncated normal form of the 1:1 resonance bifurcation, see [2, 16]. 
Several analytic and numerical studies were devoted to this (or an equivalent) map, including [3, 7, 9].

The map (1) can be approximated by the time-1 flow of a vector-field which has a BT equilibrium at $(0,0)$ (the fixed point as for the map). The corresponding ODE system is called the approximating system. The dynamic behavior of (1) is different from that of the approximating system. In the approximating system, the parameters that correspond to the saddle homoclinic bifurcation form a curve, while a homoclinic zone bounded by two curves corresponding to primary homoclinic tangencies exists in (1), see Fig 1. If parameters $\left(\nu_{1}, \nu_{2}\right)$ are located inside the homoclinic zone, then the BT map possesses transversal homoclinic orbits. On the curves of the tangencies, the homoclinic orbits become nontransversal.

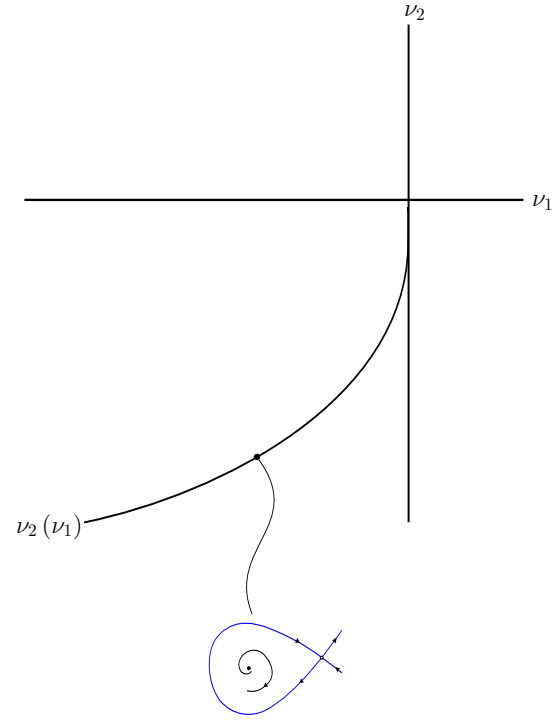

(a)

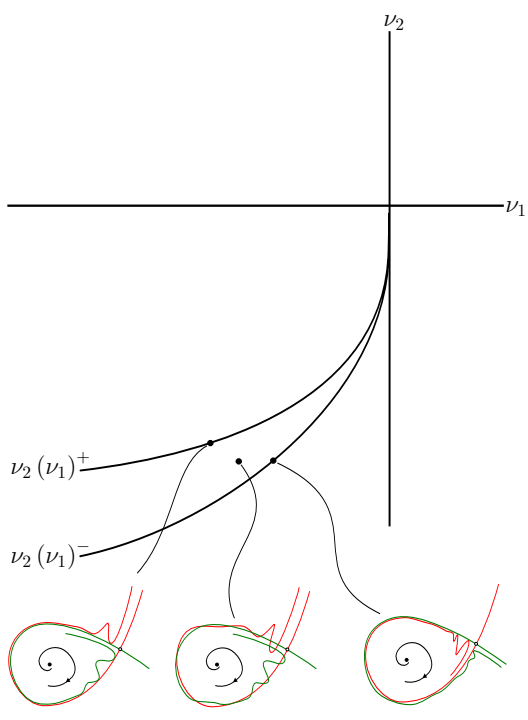

(b)

Figure 1.: The partial bifurcation diagram in the unfolding parameter space: (a) The homoclinic curve $\nu_{2}\left(\nu_{1}\right)$ of the approximating vector-field. (b) The homoclinic structure of the BT map. For points $\left(\nu_{1}, \nu_{2}\right)$ located between the lower and the upper curves (i.e., $\nu_{2}\left(\nu_{1}\right)^{+}$and $\left.\nu_{2}\left(\nu_{1}\right)^{-}\right)$, transversal homoclinic orbits exist. These orbits collide on the curves of tangencies $\nu_{2}\left(\nu_{1}\right)^{+}, \nu_{2}\left(\nu_{1}\right)^{-}$.

A numerical method to continue branches of homoclinic orbits and homoclinic tangencies, given a good starting point, was developed in [5, 6] and implemented in MATLAB [15. This algorithm consists of finding a finite number of intersection points of the stable and unstable manifolds of the saddle, i.e., the connecting orbit, by growing the manifolds from linear approximations near the saddle [8]. These points can be continued in one parameter until the limit point is detected, which corresponds to a tangency of the stable and unstable manifolds. Continuation of such a limit point in two parameters gives the homoclinic tangency curve. Therefore, if a good asymptotic for this parameter exists, one can use known numerical methods to compute the homoclinic tangency.

Although the exact bifurcation structure is different for the map (1) and the approximating vector-field, the analysis of the vector field provides information that is hardly available by considering the map alone. The approximating system allows to 
predict the homoclinic structure that appears in the map, since this structure occurs near the saddle homoclinic bifurcation in the approximating system. Our main goal throughout this paper is to derive a better asymptotic formula for the homoclinic parameters of the BT map. We compare two methods to approximate the BT map by a vector-field, namely, the interpolating technique [7, 9] and the method of Picard iterations [16, 18. We show the superiority of the asymptotic based on the second method. The new asymptotic is derived by

(a) considering all second-order terms w.r.t. coordinates and parameters in the approximating system;

(b) using the accurate homoclinic asymptotic predictor from [1, 17], instead of an incorrect asymptotic from [4] (which is also used in [19]) or a rough Melnikov approximation employed in [7, 9, 10].

The paper is organized as follows. In Section 2 we describe two methods to approximate the BT map by a vector-field. This gives two approximating systems. In Section 3 we discuss asymptotics of the homoclinic parameter in both systems. Section 4 compares both asymptotics with the actual homoclinic structure in the BT map obtained by accurate numerical continuation. The new asymptotic for the homoclinic parameters based on Picard iterations proved to be the most accurate. Moreover, we show how to use this asymptotic to compute branches of the homoclinic tangencies in the BT map.

\section{Approximation by a flow}

In this section we compare two methods used in the literature to approximate the map (1) by a vector-field, namely, the formal interpolating technique and the method of Picard iterations. The comparison demonstrates their formal equivalence.

\section{1. $\quad$ Formal interpolation method}

It is possible to formally interpolate the map $G$ by a vector-field

$$
U_{\nu}=P(u, \nu) \partial u_{0}+Q(u, \nu) \partial u_{1},
$$

where $P$ and $Q$ are formal power series in $u_{0}, u_{1}, \nu_{1}$ and $\nu_{2}$. Define the exponent,

$$
e^{U_{\nu}}=I+\sum_{n \geq 1} \frac{1}{n !} U_{\nu}^{n},
$$

where $U_{\nu}^{n}$ stands for the vector-field $U_{\nu}$ applied $n$-times. Generally speaking, we say that $U_{\nu}$ is the approximating vector-field of the map $G$ if the time- 1 shift along trajectories of $U_{\nu}$ (i.e., $\left.e^{U_{\nu}}\left(u_{0}, u_{1}\right)\right)$ coincides with $G$. Following [9], we say that the $\delta$-order of the monomial $u_{0}^{k} u_{1}^{l} \nu_{1}^{m} \nu_{2}^{n}$ is given by the following weight function:

$$
\delta\left(u_{0}^{k} u_{1}^{l} \nu_{1}^{m} \nu_{2}^{n}\right)=2 k+3 l+4 m+2 n .
$$


Thus the formal series $P$ and $Q$ can be expressed as

$$
\left\{\begin{array}{l}
P=\sum_{i \geq 3} p_{i}\left(u_{0}, u_{1}, \nu_{1}, \nu_{2}\right) \\
Q=\sum_{j \geq 4} q_{j}\left(u_{0}, u_{1}, \nu_{1}, \nu_{2}\right)
\end{array}\right.
$$

where $p_{i}$ and $q_{j}$ are $\delta$-homogenous polynomials of order $i$ and $j$ respectively, i.e.,

$$
\left\{\begin{array}{l}
p_{i}=\sum_{q_{j}}=\sum_{2 k+3 l+4 m+2 n=j} c_{k l m n} u_{0}^{k} u_{1}^{l} \nu_{1}^{m} \nu_{2}^{n}, \\
d_{k l m n} u_{0}^{k} u_{1}^{l} \nu_{1}^{m} \nu_{2}^{n} .
\end{array}\right.
$$

with coefficients $c_{k l m n}, d_{k l m n} \in \mathbb{R}$ to be determined. We ignore the convergence question. Then the vector-field $U_{\nu}$ can be expanded into a sum of $\delta$-homogenous polynomial vector-fields,

$$
U_{\nu}=\sum_{i \geq 1} U_{i}, \quad U_{i}=p_{i+2} \partial u_{0}+q_{i+3} \partial u_{1}
$$

We note that when we apply $U_{i}$ to a $\delta$-homogeneous polynomial of $\delta$-order $n$ we obtain a $\delta$-homogenous polynomial of $\delta$-order $n+i$, which explains the importance of the $\delta$-ordering. Moreover, the $U_{1}$ vector-field happens to be Hamiltonian, which will be used later on. Using the assumptions above, we are ready to reproduce for completeness the proof of the following proposition from [10].

LEMma 1. For all sufficiently small $\|\nu\|$, there is a unique formal vector-field $Z$ such that

$$
(G, \nu)=e^{Z}(u, \nu)
$$

Proof. Assume that the vector-field $Z$ can be expressed as

$$
Z=P \partial u_{0}+Q \partial u_{1}+R \partial \nu_{1}+S \partial \nu_{2},
$$

where $P, Q, R$ and $S$ are formal power series in $u_{0}, u_{1}, \nu_{1}, \nu_{2}$. Expand the vector-field $Z$ into a sum of $\delta$-homogenous polynomial vector-fields

$$
Z=\sum_{i \geq 1} Z_{i}
$$

Let $\pi_{i}$ denote the projection of a formal series onto the subspace of $\delta$-homogenous 
polynomials of $\delta$-order $i \geq 1$ and define

$$
\left\{\begin{array}{l}
p_{i+2}=\pi_{i+2}\left(u_{0}+u_{1}-e^{Z} u_{0}\right), \\
q_{i+3}=\pi_{i+3}\left(u_{1}+g(u, \nu)-e^{Z} u_{1}\right), \\
r_{i+4}=\pi_{i+4}\left(\nu_{1}-e^{Z} \nu_{1}\right), \\
s_{i+2}=\pi_{i+2}\left(\nu_{2}-e^{Z} \nu_{2}\right) .
\end{array}\right.
$$

where $g(u, \nu):=\nu_{1}+\nu_{2} u_{1}+a u_{0}^{2}+b u_{0} u_{1}$. The right hand side of (9) are finite sums and depend on $p_{n_{1}}$ with $3 \leq n_{1} \leq i-2, q_{n_{2}}$ with $4 \leq n_{2} \leq i-1, r_{n_{3}}$ with $5 \leq n_{3} \leq i$ and $s_{n_{4}}$ with $3 \leq n_{4} \leq i-2$ as well as on the coefficients of the terms of $(G, \nu)$. Define the equality

$$
Z u_{0}=\sum_{i \geq 3} p_{i}, \quad Z u_{1}=\sum_{i \geq 4} q_{i}, \quad Z \nu_{1}=\sum_{i \geq 5} r_{i}, \quad Z \nu_{2}=\sum_{i \geq 3} s_{i} .
$$

Then by taking the leading order in (9), we obtain

$$
Z_{1}(u, \nu)=\left(p_{3}, q_{4}, r_{5}, s_{3}\right)=\left(u_{1}, \nu_{1}+a u_{0}^{2}, 0,0\right) .
$$

The polynomials $p_{3}, q_{4}, r_{5}$ and $s_{3}$ are uniquely defined and hence the recurrent polynomials $p_{i+2}, q_{i+3}, r_{i+4}$ and $s_{i+2}, i \geq 2$ are also uniquely defined. Also, it is clear that the polynomials $r_{i+4}, s_{i+2}$ are equal to zero for all $i \geq 2$. Thus we can write the $\delta$-homogenous polynomials vector-field $Z_{i}, i \geq 2$ as follows:

$$
Z_{i}=\left(U_{i}, 0\right)
$$

where

$$
U_{i}=p_{i+2} \partial u_{0}+q_{i+3} \partial u_{1}, \quad i \geq 2 .
$$

This vector-field $Z$ satisfies (8).

With a suitable number of terms in (3) and solving (9) for $i \geq 2$, we obtain

$$
\begin{aligned}
U_{1} & =u_{1} \partial u_{0}+\left(\nu_{1}+a u_{0}^{2}\right) \partial u_{1}, \\
U_{2} & =-\frac{1}{2}\left(\nu_{1}+a u_{0}^{2}\right) \partial u_{0}+\left(\nu_{2} u_{1}+(b-a) u_{0} u_{1}\right) \partial u_{1}, \\
U_{3} & =\left(-\frac{1}{2} \nu_{2} u_{1}+\left(\frac{2}{3} a-\frac{1}{2} b\right) u_{0} u_{1}\right) \partial u_{0}+ \\
& \left(-\frac{1}{2}\left(\nu_{1} \nu_{2}+a \nu_{2} u_{0}^{2}\right)+\frac{1}{2}\left(\frac{1}{3} a-b\right) u_{1}^{2}+\left(\frac{2}{3} a-\frac{1}{2} b\right) \nu_{1} u_{0}+\left(a^{2}-\frac{1}{2} a b\right) u_{0}^{3}\right) \partial u_{1},
\end{aligned}
$$


It follows from Lemma 1 that the map (1) can be formally interpolated by the autonomous vector-field

$$
U_{\nu}=U_{1}+U_{2}+U_{3}+\ldots
$$

\subsection{The method of Picard iterations}

Following [16], we start with writing (1) near the fixed point as a 4-dimensional map

$$
(G, \nu):(u, \nu) \mapsto A(u, \nu)+\left(F_{\nu}^{(2)}, 0\right)
$$

where

$$
A=\left(\begin{array}{cccc}
1 & 1 & 0 & 0 \\
0 & 1 & 1 & 0 \\
0 & 0 & 1 & 0 \\
0 & 0 & 0 & 1
\end{array}\right), \quad F_{\nu}^{(2)}:=\left(\begin{array}{c}
0 \\
\nu_{2} u_{1}+a u_{0}^{2}+b u_{0} u_{1}
\end{array}\right)
$$

Assume that the approximating system to (13) has the same equilibrium (i.e., the fixed point of (1)), and can be written as

$$
(\dot{u}, \dot{\nu})=\Lambda(u, \nu)+\left(f_{\nu}^{(2)}(u, \nu), 0\right)
$$

where $\Lambda$ is a $4 \times 4$ matrix and the components of the two-dimensional vector $f_{\nu}^{(2)}(u)$ are smooth polynomials of order 2 in $u_{0}, u_{1}, \nu_{1}$ and $\nu_{2}$ with coefficients to be determined. The flow $\varphi_{\nu}^{t}(u)$ generated from the component $\left(\dot{u}_{0}, \dot{u}_{1}\right)$ in 14 can be seen as the first two components of the flow

$$
(u, \nu) \mapsto \phi_{\nu}^{t}(u)
$$

generated by 14 , i.e., $\phi_{\nu}^{t}(u):=\left(\varphi_{\nu}^{t}(u), \nu\right)^{\mathrm{T}}$. The method of Picard iterations [18] can be used to approximate the flow map (15). If the corresponding terms in the generated time-1 flow, i.e., $\phi_{\nu}^{1}(u)$ and $\sqrt{13}$ coincide, then system 14 is said to be the approximating system of the map (13). The solution of the linear part of (14) can be used as initial data for the Picard iterations. Therefore, we set

$$
U^{0}(t)=e^{\Lambda t}(u, \nu) .
$$

Since we seek a flow whose time-1 orbits coincide with (13), we have

$$
e^{\Lambda}=A .
$$

Solving for $\Lambda$ gives

$$
\Lambda=\left(\begin{array}{cccc}
0 & 1 & -\frac{1}{2} & 0 \\
0 & 0 & 1 & 0 \\
0 & 0 & 0 & 0 \\
0 & 0 & 0 & 0
\end{array}\right)
$$


Now, we perform a Picard iteration to compute the second order terms to $\phi^{1}$ :

$$
U^{1}(t)=e^{\Lambda t}(u, \nu)^{\mathrm{T}}+\int_{0}^{t} e^{\Lambda(t-\tau)}\left(f_{\nu}^{(2)}\left(U^{0}(\tau)\right), 0\right) d \tau .
$$

Comparing the coefficients of the similar terms in (17) for $t=1$ and 13 specifies the components of $f_{\nu}^{(2)}(u)$. Thus, we have the following extension of Lemma 9.8 from [16, Sec.9.5.2].

Lemma 2. For all sufficiently small $\|\nu\|$, the map (1) can be represented as

$$
u \mapsto \varphi_{\nu}^{1}(u)+\mathcal{O}\left(\|(u, \nu)\|^{3}\right)
$$

where $\varphi_{\nu}^{t}(u)$ is the flow of a planar system

$$
\dot{u}=\left(\begin{array}{ll}
0 & 1 \\
0 & 0
\end{array}\right) u+\left(\begin{array}{c}
-\frac{1}{2} \nu_{1} \\
\nu_{1}
\end{array}\right)+f_{\nu}^{(2)}(u)
$$

where

$$
f_{\nu}^{(2)}(u)=\left(\begin{array}{c}
\xi_{00}(\nu) \\
\zeta_{00}(\nu)
\end{array}\right)+\left(\begin{array}{l}
\xi_{10}(\nu) u_{0}+\xi_{01}(\nu) u_{1} \\
\zeta_{10}(\nu) u_{0}+\zeta_{01}(\nu) u_{1}
\end{array}\right)+\left(\begin{array}{c}
\frac{1}{2} \xi_{20} u_{0}^{2}+\xi_{11} u_{0} u_{1}+\frac{1}{2} \xi_{02} u_{1}^{2} \\
\frac{1}{2} \zeta_{20} u_{0}^{2}+\zeta_{11} u_{0} u_{1}+\frac{1}{2} \zeta_{02} u_{1}^{2}
\end{array}\right)
$$

with

$$
\begin{array}{ll}
\xi_{00}(\nu)=\frac{1}{20}(2 b-a) \nu_{1}^{2}+\frac{1}{3} \nu_{1} \nu_{2}, & \zeta_{00}(\nu)=\left(\frac{1}{30} a-\frac{1}{12} b\right) \nu_{1}^{2}-\frac{1}{2} \nu_{1} \nu_{2}, \\
\xi_{10}(\nu)=\left(\frac{1}{3} b-\frac{1}{2} a\right) \nu_{1}, & \zeta_{10}(\nu)=\left(\frac{2}{3} a-\frac{1}{2} b\right) \nu_{1} \\
\xi_{01}(\nu)=\left(\frac{1}{5} a-\frac{5}{12} b\right) \nu_{1}-\frac{1}{2} \nu_{2}, & \zeta_{01}(\nu)=\left(\frac{1}{2} b-\frac{1}{6} a\right) \nu_{1}+\nu_{2}, \\
\xi_{20}=-a, & \zeta_{20}=2 a, \\
\xi_{11}=\left(\frac{2}{3} a-\frac{1}{2} b\right), & \zeta_{11}=(b-a), \\
\xi_{02}=\left(\frac{2}{3} b-\frac{1}{3} a\right), & \zeta_{02}=\frac{1}{3} a-b .
\end{array}
$$

Note that, if we reorder the terms of $(19)$ according to (4), then up to the quadratic terms in $(u, \nu)$, the corresponding terms of the systems (12) and 190 coincide. Adding further steps in the interpolation method and in the Picard iterations will give us:

$$
\sum_{i=1}^{\infty} U_{i} \equiv\left(\begin{array}{ll}
0 & 1 \\
0 & 0
\end{array}\right) u^{\mathrm{T}}+\left(\begin{array}{c}
-\frac{1}{2} \nu_{1} \\
\nu_{1}
\end{array}\right)+\sum_{i=2}^{\infty} f_{\nu}^{(n)}(u)
$$

Thus the interpolating technique and the method of Picard iteration are equivalent. Nevertheless, truncations to the same iteration are different in the two methods, leading to different predictors. 


\section{The homoclinic asymptotic}

In this section we derive an improved asymptotic formula for the borders of the homoclinic zone in the BT map.

\subsection{An asymptotic based on Melnikov integral}

First we proceed as in 9 and consider the vector-field (12). The first order vector-field $U_{1}$ defines a Hamiltonian system with the energy function

$$
H=\frac{1}{2} \dot{u}_{0}^{2}+V\left(u_{0}, \nu_{1}\right)-k, \quad k \in \mathbb{R},
$$

where $\dot{u}_{0}=u_{1}$ and the function $V\left(u_{0}, \nu_{1}\right)$ is given by

$$
V\left(u_{0}, \nu_{1}\right)=-\int_{0}^{u_{0}}\left(\nu_{1}+a u^{2}\right) d u=-\left(\nu_{1} u_{0}+\frac{a u_{0}^{3}}{3}\right) .
$$

If $\frac{-\nu_{1}}{a} \geq 0$ then equation 20 has a homoclinic loop defined by $k=\frac{2}{3} \sqrt{\frac{\left(-\nu_{1}\right)^{3}}{a}}$. The function $V\left(u_{0}, \nu_{1}\right)$ and the phase portrait of the homoclinic solution of (20) are shown in Fig.2 The solution curve in the $\left(u_{0}, u_{1}\right)$-plane satisfies the homoclinic condition i.e., the phase point $\left(u_{0}, u_{1}\right)$ approaches the saddle point

$$
\left(u_{0}^{s}, u_{1}^{s}\right)=\left(\sqrt{\frac{-\nu_{1}}{a}}, 0\right), \operatorname{sign}\left(\nu_{1}\right)=-\operatorname{sign}(a),
$$

as $t \rightarrow \pm \infty$. The related homoclinic solution can be found explicitly

$$
L_{0}(t)=\left(u_{0}(t), u_{1}(t)\right)=\left(\sqrt{\frac{-\nu_{1}}{a}}\left(1-3 \operatorname{sech}^{2}\left(t \frac{\sqrt[4]{-a \nu_{1}}}{\sqrt{2}}\right)\right), \frac{d}{d t} u_{0}(t)\right) .
$$

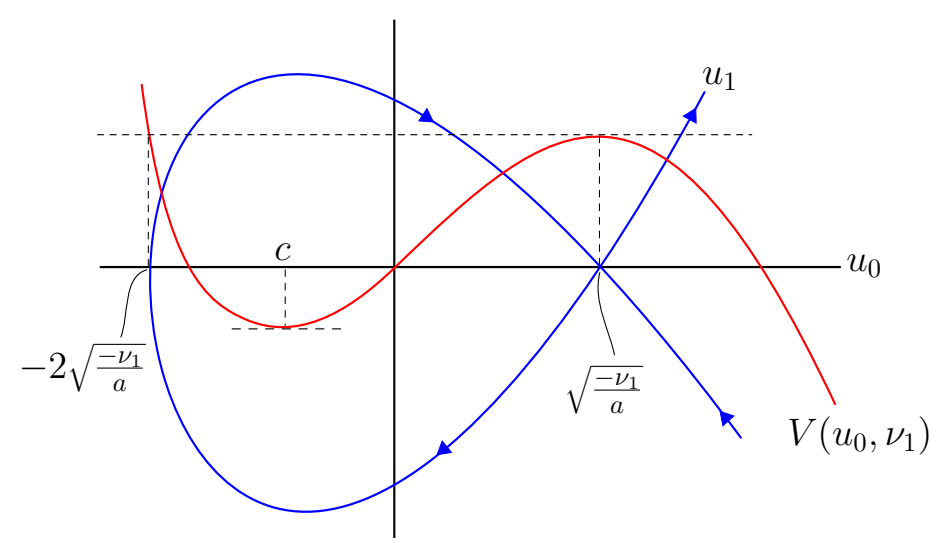

Figure 2.: The function $V\left(u_{0}, \nu_{1}\right)$ and the phase portrait of equation 200 . 
This solution persists for $U_{\nu} \approx U_{1}+U_{2}+U_{3}$ if the Melnikov integral

$$
\begin{aligned}
M(\nu) & =\left.\int_{-\infty}^{\infty}\left(U_{2} h+U_{3} h\right)\right|_{L_{0}(t)} d t \\
& =-\frac{24}{7} \frac{(b-2 a)}{a} \nu_{1}^{2}+\frac{24}{5 \sqrt{a}}\left(-\nu_{1}\right)^{\frac{3}{2}} \nu_{2}
\end{aligned}
$$

vanishes [13]. The function $M(\nu)$ has zero along the curve

$$
\nu_{2}^{M}=\frac{5}{7 \sqrt{a}}(b-2 a) \sqrt{-\nu_{1}},
$$

which gives a rough asymptotic for the homoclinic curve in the parameter space obtained in [9].

\subsection{Asymptotic based on Picard iteration and a higher-order prediction}

It is clear that system $(19)$ has a BT point $u=0$ at $\nu=0$. The Jacobian matrix of (19) evaluated at the BT point is

$$
A=\left(\begin{array}{ll}
0 & 1 \\
0 & 0
\end{array}\right)
$$

At the BT point, the Taylor expansion of the R.H.S. of $(19)$ can be expressed as

$$
\dot{u}=A u+J_{1} \nu+\frac{1}{2} B(u, u)+A_{1}(u, \nu)+\frac{1}{2} J_{2}(\nu, \nu)+\frac{1}{6} C(u, u, u)+\frac{1}{2} B_{1}(u, u, \nu)+\ldots
$$

where

$$
\begin{aligned}
& J_{1}=\left(\begin{array}{cc}
-\frac{1}{2} & 0 \\
1 & 0
\end{array}\right), \quad B(u, v)=\left(\begin{array}{c}
\left(\frac{2}{3} a-\frac{1}{2} b\right)\left(u_{1} v_{0}+u_{0} v_{1}\right)+\left(\frac{2}{3} b-\frac{1}{3} a\right) u_{1} v_{1}-a u_{0} v_{0} \\
(b-a)\left(u_{1} v_{0}+u_{0} v_{1}\right)+\left(\frac{1}{3} a-b\right) u_{1} v_{1}+2 a u_{0} v_{0}
\end{array}\right), \\
& A_{1}(u, \nu)=\left(\begin{array}{c}
\left(\frac{1}{3} b-\frac{1}{2} a\right) u_{0} \nu_{1}+\left(\frac{1}{5} a-\frac{5}{12} b\right) u_{1} \nu_{1}-\frac{1}{2} u_{1} \nu_{2} \\
\left(\frac{2}{3} a-\frac{1}{2} b\right) u_{0} \nu_{1}+\left(\frac{1}{2} b-\frac{1}{6} a\right) u_{1} \nu_{1}+u_{1} \nu_{2}
\end{array}\right) \\
& J_{2}(\nu, \mu)=\left(\begin{array}{c}
\left(\frac{1}{5} b-\frac{1}{10} a\right) \nu_{1} \mu_{1}+\frac{1}{3} \nu_{2} \mu_{1}+\frac{1}{3} \nu_{1} \mu_{2} \\
\left(\frac{1}{15} a-\frac{1}{6} b\right) \nu_{1} \mu_{1}-\frac{1}{2} \nu_{2} \mu_{1}-\frac{1}{2} \nu_{1} \mu_{2}
\end{array}\right), \quad B_{1}=C=\left(\begin{array}{c}
0 \\
0
\end{array}\right) .
\end{aligned}
$$

The homoclinic solution of 25 then can be computed by the method described in [17, Appendix B]. The homoclinic parameter of 25) is found as

$$
\left(\begin{array}{c}
\nu_{1} \\
\nu_{2}
\end{array}\right)=\frac{\varepsilon^{2}}{a}\left(\begin{array}{c}
0 \\
\frac{10}{7}(b-2 a)
\end{array}\right)-\frac{\varepsilon^{4}}{a}\left(\begin{array}{c}
4 \\
\delta
\end{array}\right)+\mathcal{O}\left(\varepsilon^{5}\right), \quad 0<\varepsilon \ll 1,
$$

where

$$
\delta:=\frac{1}{2401 a^{2}}(b-2 a)\left(857 a^{2}-3650 a b-288 b^{2}\right)+\frac{2 a^{2}-5 a b+b^{2}}{a} .
$$


Using

$$
\varepsilon \approx \sqrt[4]{\frac{a\left(-\nu_{1}\right)}{4}},
$$

we obtain the following improved approximation for the homoclinic bifurcation curve of 19$)$ in the parameter space $\left(\nu_{1}, \nu_{2}\right)$ :

$$
\nu_{2}=\frac{5}{7 \sqrt{a}}(b-2 a) \sqrt{-\nu_{1}}+\frac{1}{4} \delta \nu_{1}+\mathcal{O}\left(\left|\nu_{1}\right|^{\frac{5}{4}}\right),
$$

where $\delta$ is defined by $(27)$. Note that the first term in 29 coincides with $\nu_{2}^{M}$ from (24), as one could expect.

\section{The homoclinic zone of the Bogdanov-Takens map}

To check whether the homoclinic asymptotic parameters 24 and 29) are located inside the homoclinic zone of (1), we use the MATLAB interactive toolbox for numerical study of smooth maps MatContM to compute the stable and unstable manifolds of the saddle at the approximated homoclinic parameter. MatContM uses an algorithm originally adopted from [8] (for details on the algorithm used see [14]). We set $a=b=1$ and $\nu_{1}=-0.15$. Then we use the saddle fixed point (21) and the asymptotics of the homoclinic parameter (24), (29) to obtain $\left(u_{0}^{s}, u_{1}^{s}\right)=(0.387298,0)$ and $\nu_{2}=-0.249762, \nu_{2}^{M}=-0.276642$. The grown stable and unstable manifolds of the saddle $\left(u_{0}^{s}, u_{1}^{s}\right)$ at $\left(\nu_{1}, \nu_{2}^{M}\right)$ and $\left(\nu_{1}, \nu_{2}\right)$ are shown in Fig $3(\mathrm{a})$ and Fig $3(\mathrm{~b})$, respectively. For $\nu_{1}=-0.15$, it is clear that the predicted homoclinic parameter based on (24) is located outside the homoclinic zone of (1). The result is not surprising because 24 is derived by the Melnikov method which gives the zero-order approximation for the homoclinic parameter. In Fig $3(\mathrm{~b})$ the stable and unstable manifolds of the saddle $\left(u_{0}^{s}, u_{1}^{s}\right)$ intersect transversally. This immediately indicates the usefulness of the new asymptotic 29).

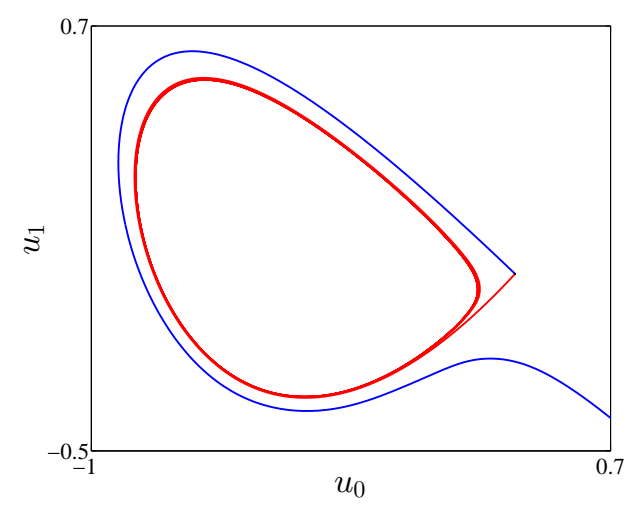

(a)

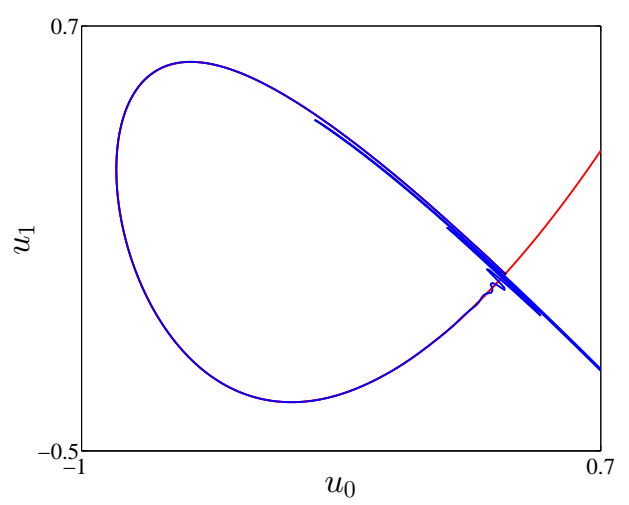

(b)

Figure 3.: The grown stable and unstable manifolds of (1) for $a=b=1, \nu_{1}=-0.15$, $\left(u_{0}^{s}, u_{1}^{s}\right)=(0.387298,0)$ and (a) $\nu_{2}^{M}=-0.276642$, (b) $\nu_{2}=-0.249762$. 
Using MatContM we compute the intersection points of the manifolds presented in Fig $3 \mathrm{~b}$. These points are continued in one parameter $\left(\nu_{1}\right.$ freed while $\nu_{2}$ is fixed) until two limit points are detected, which correspond to tangencies of the stable and unstable manifolds, see Fig.4(a) Next, Fig 4(b) and 4(c) show the corresponding tangential homoclinic orbit in the state space at the limit points $\mathrm{LP}_{1}$ and $\mathrm{LP}_{2}$ in Fig $4(\mathrm{a})$ respectively. Continuation of such limit points in two parameters $\left(\nu_{1}, \nu_{2}\right)$ gives the full homoclinic tangencies structure shown in Fig.4(d).

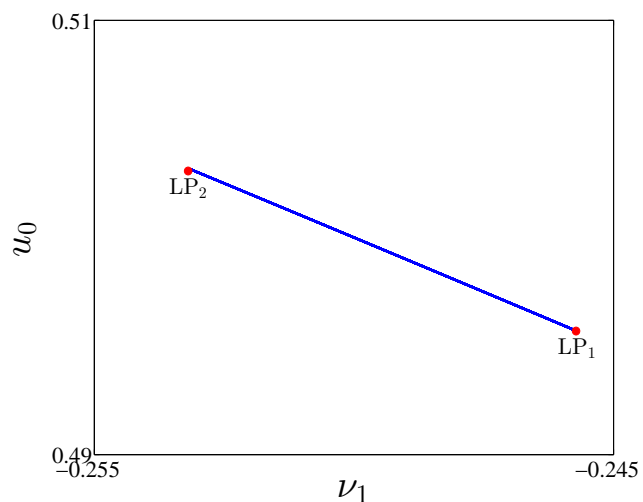

(a)

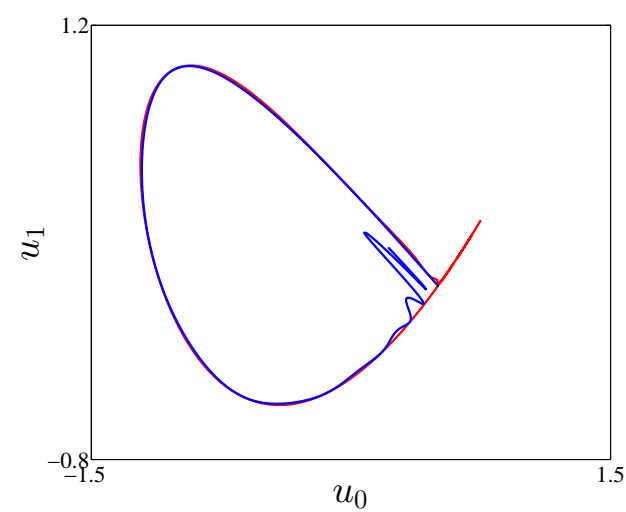

(c)

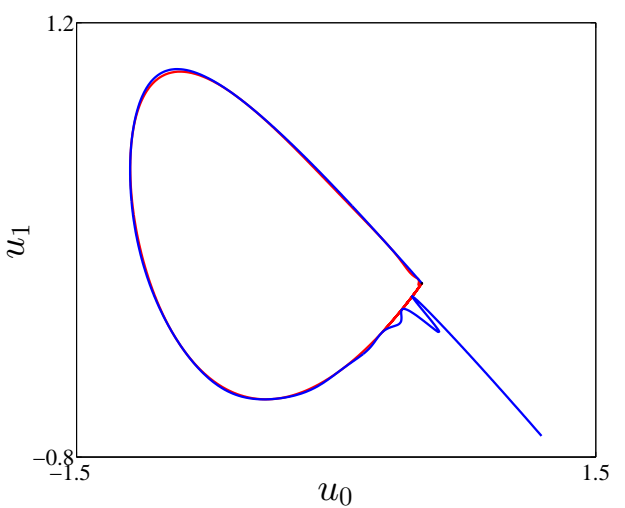

(b)

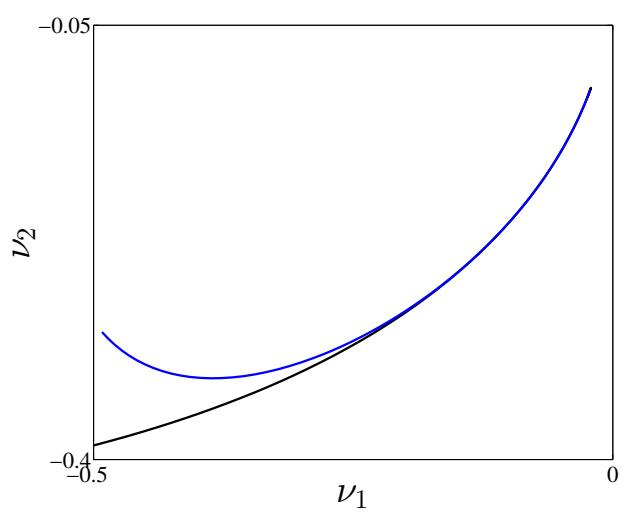

(d)

Figure 4.: (a) The limit points are computed by continuing the homoclinic points in Fig $3 \mathrm{~b}$. During continuation, $\nu_{1}$ is freed while $\nu_{2}$ is fixed, (b) Stable and unstable manifolds along the first homoclinic tangential point (i.e., $\mathrm{LP}_{1}$ ), (c) Stable and unstable manifolds along the second homoclinic tangential point (i.e., $\mathrm{LP}_{2}$ ), (d) Two branches of the tangential homoclinic orbits are computed by continuing both of the LP's on Fig.4 a with $\nu_{1}$ and $\nu_{2}$ free. Upper (blue) is $\mathrm{LP}_{2}$, lower (black) is $\mathrm{LP}_{1}$.

\section{Discussion}

Since we now have the whole homoclinic structure in the BT map, we can compare the numerically computed tangency branches with the asymptotic of the homoclinic curve (29) and the homoclinic curve obtained by (24) (see also [7, 11]), see Fig.5. This comparison demonstrates the advantage of the asymptotic 29$]$ for bigger $\left|\nu_{1}\right|$. 


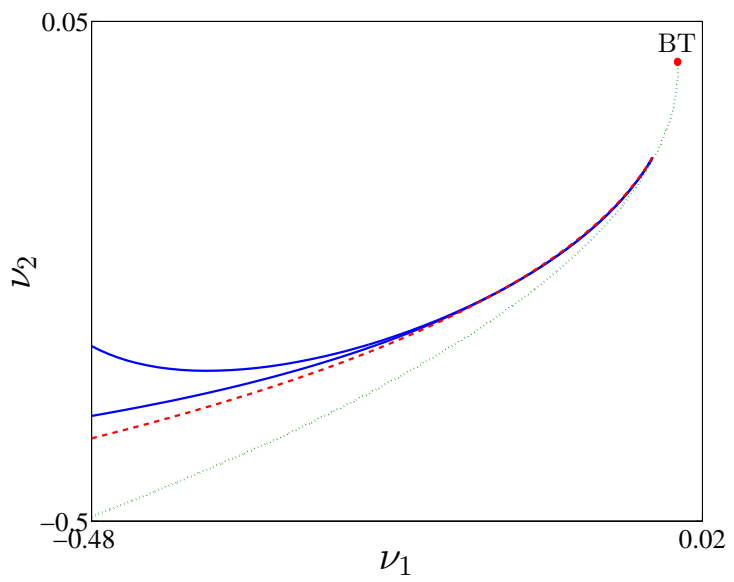

Figure 5.: Numerically computed branches of homoclinic tangencies (solid blue curves, the upper is LP2, the lower is LP1) of (1) are compared with: the asymptotic of the homoclinic curve (29) (dashed curve) and the rough approximation (24) (dotted curve). The advantage of the proposed asymptotic is evident.

Far away from the BT point, the predicted curve is not located in the homoclinic zone (i.e., between the homoclinic tangencies). When we approach the BT point the prediction enters the homoclinic zone and we believe that it stays there.

We have observed that accounting for cubic terms in the approximating system (14) via one extra Picard iteration does not improve the accuracy of the prediction but rather worsen it. This could be related to the fact that already the second Picard iterate employed in Lemma 2 coincides with the BT map (1). Further investigation of this phenomenon is required. Note also that the numerically computed homoclinic tangency curves deviate from the "exact" ones due to the approximate boundary conditions used.

The obtained asymptotic (29) predicts only the location of the homoclinic wedge in the parameter plane. The problem to predict a homoclinic orbit in the BT map is much more involved, since the time-1 map of the approximating ODE system at homoclinic parameter values has a continuous family of such orbits, while only two primary homoclinic orbits exist in the map. A possible tool to deal with this problem is the Melnikov function for planar maps [12]. This function has to be evaluated along the homoclinic orbit of the approximating system, and its zeroes provide the asymptotic position of the homoclinic orbits as intersections of the stable and unstable invariant manifolds of the saddle. In principle, this allows to approximate both transverse and nontransverse homoclinic orbits. This issue was ignored in [19, where an arbitrary point of the homoclinic orbit in the approximating system was taken as a point in the homoclinic orbit of the map.

The analysis of the BT map is the first step towards developing a robust predictor for homoclinic orbits bifurcating from a 1:1 resonance fixed point in generic $n$-dimensional smooth maps. Such a predictor should combine correct asymptotics of the bifurcating homoclinic orbits in the normal form with the parameter-dependent reduction to the two-dimensional center manifold. The former would probably require to add some extra terms to the BT map (1), while the latter can easily be done using the homological equation technique applied in the ODE-case in [1, 17]. 


\section{Acknowledgment}

The authors are thankful to Dr. H.G.E. Meijer (University of Twente, The Netherlands) for useful discussions.

\section{References}

[1] B. Al-Hdaibat, W. Govaerts, Yu. A. Kuznetsov, and H. G. E. Meijer. Initialization of homoclinic solutions near Bogdanov-Takens points: Lindstedt-Poincaré compared with regular perturbation method. To appear in SIAM J. Appl. Dynamical Systems, 2016.

[2] V. I. Arnold. Geometrical Methods in the Theory of Ordinary Differential Equations. Springer-Verlag, 1983.

[3] D. K. Arrowsmith, J. H. E. Cartwright, A. N. Lansbury, and C. M. Place. The Bogdanov map: bifurcations, mode locking, and chaos in a dissipative system. International Journal of Bifurcation and Chaos, 03(04):803-842, 1993.

[4] W.-J. Beyn. Numerical analysis of homoclinic orbits emanating from a TakensBogdanov point. IMA J. Numer. Anal., 14(3):381-410, 1994.

[5] W.-J. Beyn, T. Hüls, and Y. Zou. Numerical analysis of degenerate connecting orbits for maps. International Journal of Bifurcation and Chaos, 14(10):3385-3407, 2004.

[6] W.-J. Beyn and J.-M. Kleinkauf. The numerical computation of homoclinic orbits for maps. SIAM J. Numer. Anal, 34:1207-1236, 1996.

[7] H. Broer, R. Roussarie, and C. Simó. Invariant circles in the Bogdanov-Takens bifurcation for diffeomorphisms. Ergodic Theory and Dynamical Systems, 16:1147-1172, 12 1996.

[8] J. England, B. Krauskopf, and H. Osinga. Computing one-dimensional stable manifolds and stable sets of planar maps without the inverse. SIAM Journal on Applied Dynamical Systems, 3(2):161-190, 2004.

[9] V. Gelfreich. Chaotic zone in the Bogdanov-Takens bifurcation for diffeomorphisms. In H. G. W. Begehr, R. P. Gilbert, and M. W. Wong, editors, Analysis and Applications - ISAAC 2001, volume 10 of International Society for Analysis, Applications and Computation, pages 187-197. Springer, US, 2003.

[10] V. Gelfreich and V. Naudot. Analytic invariants associated with a parabolic fixed point in $x \in \mathbb{C}^{2}$. Ergodic Theory and Dynamical Systems, 28:1815-1848, 122008.

[11] V. Gelfreich and V. Naudot. Width of homoclinic zone for quadratic maps. Experimental Mathematics, 18(4):409-427, 2009.

[12] M. L. Glasser, V. G. Papageorgiou, and T. C. Bountis. Mel'nikov's function for twodimensional mappings. SIAM J. Appl. Math., 49(3):692-703, 1989.

[13] J. Guckenheimer and P. Holmes. Nonlinear Oscillations, Dynamical Systems and Bifurcations of Vector Fields. Springer-Verlag, New York, 1983.

[14] R. Khoshsiar Ghaziani. Bifurcations of maps: numerical algorithms and applications. $\mathrm{PhD}$ thesis, Ghent University, 2008.

[15] R. Khoshsiar Ghaziani, W. Govaerts, Yu.A. Kuznetsov, and H. G. E. Meijer. Numerical continuation of connecting orbits of maps in MATLAB. Journal of Difference Equations and Applications, 15:849-875, 2009.

[16] Yu. A. Kuznetsov. Elements of Applied Bifurcation Theory. Springer-Verlag, New York, 3rd edition, 2004.

[17] Yu. A. Kuznetsov, H. G. E. Meijer, B. Al-Hdaibat, and W. Govaerts. Accurate approximation of homoclinic solutions in Gray-Scott kinetic model. International Journal of Bifurcation and Chaos, 25(9):1550125 (10 pages), 2015.

[18] Ju.I. Neimark. The Method of Point Transformations in the Theory of Nonlinear Oscillations. Nauka, Moscow, 1972. [in Russian]. 
